\title{
ROV Assisted Magnetic Induction Communication Field Tests in Underwater Environments
}

\author{
Debing Wei \\ University of Houston \\ dwei3@uh.edu \\ Aaron T. Becker \\ University of Houston \\ atbecker@uh.edu
}

\author{
Steban S. Soto \\ University of Houston \\ sssoto@uh.edu
}

\author{
Li Wang \\ Beijing University of Posts and \\ Telecommunications \\ liwang@bupt.edu.cn
}

\author{
Javier Garcia \\ University of Houston \\ jgarciagonzalez@uh.edu \\ Miao Pan \\ University of Houston \\ mpan2@uh.edu
}

\begin{abstract}
Magnetic Induction (MI) is a promising technique for near-field wireless underwater communications. Although the literature has some theoretical analyses and lab experiments for underwater MI communication, there is a lack of field tests in underwater environments, especially in subsea environments. In this paper, we leverage the remotely operated vehicle (ROV) and the remotely controlled boat (RCB) to develop an MI wireless communication system, and conduct field tests for MI communication performance in both fresh water and sea water. The experiment results show that even in the most challenging subsea environment, the MI communication has very good near-field transmission performance with a small coil antenna and low power consumption.
\end{abstract}

\section{KEYWORDS}

Underwater Wireless Communications; Magnetic Induction; ROV; Subsea Environment.

\section{INTRODUCTION}

Underwater wireless communications are the enabling technologies for numerous subsea applications such as underwater environment monitoring, underwater resource exploration, offshore infrastructure protection, and oceanographic data collection [4]. However, the physical signals commonly used for terrestrial wireless communications, e.g. optical signals and radio frequency (RF) electromagnetic signals, do not work well in sea water. Their poor performance is mainly because optical signals suffer from strong scattering effects and high-frequency electromagnetic waves attenuate significantly in the conductive sea water. Acoustic signals propagate well in water. However, acoustic signals are not ideal for fast underwater wireless communications because the sound propagation speed is low (roughly $1500 \mathrm{~m} / \mathrm{s}$ in water) and the available communication bandwidth is limited, since it is difficult to make a high frequency

Permission to make digital or hard copies of all or part of this work for personal or classroom use is granted without fee provided that copies are not made or distributed for profit or commercial advantage and that copies bear this notice and the full citation on the first page. Copyrights for components of this work owned by others than ACM must be honored. Abstracting with credit is permitted. To copy otherwise, or republish to post on servers or to redistribute to lists, requires prior specific permission and/or a fee. Request permissions from permissions@acm.org.

WUWNet'18, December 3-5, 2018, Shenzhen, China

(C) 2018 Association for Computing Machinery.

ACM ISBN 978-1-4503-6193-4/18/12 _.\$15.00

https://doi.org/10.1145/3291940.3291988 acoustic signal (ultrasound is only in $\mathrm{kHz}$ ). Besides, man-made underwater noise may have harmful impacts on marine mammals $[3,9,10]$. As a result, underwater wireless communications have seen less investments of time and infrastructure compared with their terrestrial counterparts, which are evolving into 5G.

Recently, magnetic induction (MI) technology has been applied in many wireless applications such as wireless power transfer [5, 8, 12] and near field wireless communications [1,2]. MI communications is considered to be a great alternative in harsh environments like underground and underwater $[2,13]$. The main reasons behind this can be summarized as follows. First, unlike RF communications, MI technology only relies on the near field component of the magnetic field to transfer information. Since the permeability of most mediums are almost the same, MI channel conditions are much more stable in comparison to RF or acoustic channels. Second, since MI systems work under relatively low frequencies, less than $\mathrm{MHz}$, signal attenuation is much slower in comparison to RF signals. Third, in comparison to acoustic signals which typically work under 100 $\mathrm{kHz}$, the operating frequency for MI systems is much higher (100 times). This means that the available communication bandwidth is much wider for MI systems than acoustic systems, meaning that the communication data rate of the former is potentially much higher than the latter. Fourth, the antenna for MI system are simple wire coils, which are economic compared to the transducer needed for acoustic systems. Finally, coil antennas can be designed as any shape and in any size, unlike RF antennas where the antenna size is determined by the operating frequencies.

Many works have focused on the channel modeling of MI communications. For example, in [13] and [6], Sun et al. and Guo et al. studied the MI channel based on the equivalent circuit and path loss model for underground and underwater environments, respectively. Wei et al. in[15] analyzed the maximum transmission range and power efficiency for subsea MI communications based on magnetic field strength and signal to noise ratio analysis. Wei et al. further proposed a geometry-conformal MI antenna that was specifically designed for autonomous underwater vehicles (AUV) and studied the corresponding MI channel capacities in [16]. All of these works $[6,13,15,16]$ focused on the theoretical aspect of MI. The realworld performance for MI communications underwater, especially in subsea environments, is still an underexplored area. Jimenez et al. measured the MI signal attenuation between two loop coils in subsea environments in [7], but do not consider the resonance coupling between two coil antennas. Wang et al. measured a MI 
link in a seawater pool with the deployment distance of $75 \mathrm{~cm}[14]$ In comparison to previous work, in this paper, we will measure the signal attenuation between two resonance coupling coil antennas under different distances in subsea environments.

In addition to the advances on MI communications, underwater robotics have also evolved significantly, allowing the performance of increasingly complex tasks. These include exploration and mapping, deployment and retrieval of sensor nodes and other types of equipment, observation of marine life, etc. With the expansion of the knowledge pool in this particular area of robotics, as well as the increasing accessibility of commercial platforms, it has become feasible to acquire and modify underwater robots for flexible research purposes. Recognizing this, we are directing our approach toward robotic systems that can carry out the wireless communications schemes autonomously.

To bridge theoretical analyses and real-world experiments of MI based wireless underwater communications, in this paper, we leverage the remotely operated vehicle (ROV) and the remotely controlled boat (RCB) to develop an MI wireless communication system. A series of sea tests were carried out in La Porte, Texas. We measured the MI communication performance such as the attenuation, transmission range.

The rest of the paper is organized as follows. Section 2 summarizes the MI channel model and describes the key parameters of the proposed experimental MI communication system. In Section 3, we describe our experiment platform. The testing results are shown in Section 4. Finally, we conclude the paper in Section 5 and provide directions for future research.

\section{PHYSICAL LAYER MODEL}

In this section, we will summarize the MI wireless channel model according to our previous work [15]. Based on this channel model, the parameters of MI wireless communication system will be determined.

\subsection{Channel Model}

Fig. 1 shows the model of a MI wireless communication system with two coil antennas. The exciting current $I_{0}$ in the transmitting coil will generate a magnetic field. The magnetic flux that passes through the receiving coil is represented by $\Phi$. The induced voltage $U_{\Phi}$ in the receiving coil is proportional to the changing rate of $\Phi$, i.e.,

$$
U_{\Phi}=-N \frac{d \Phi}{d t}
$$

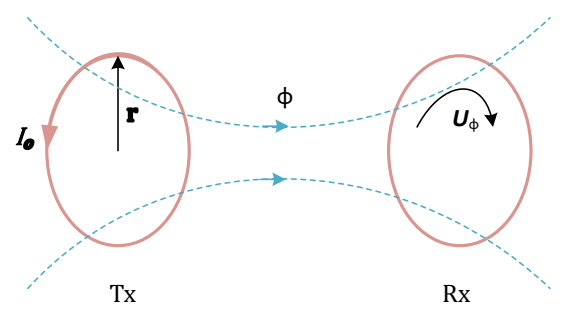

Figure 1: MI wireless communication system model. where $N$ is the number of turns of the coil antenna.

The whole system will be working on the resonance mode. Under resonance condition the induced voltage $U_{\Phi}$ will be amplified such that

$$
U_{R x}=Q U_{\Phi},
$$

where $Q$ is the quality factor of the coil antenna, and $U_{R x}$ is the received signal on the receiver side.

The signal to noise ratio (SNR) can be estimated by

$$
\mathrm{SNR}=\left(\frac{U_{R x}}{U_{n}}\right)^{2},
$$

where $U_{n}$ is the noise voltage level.

After the SNR was obtained, the system performances such as the channel capacity $C$, and bit error rate $P_{e}$ can be evaluated based on the Shannon capacity theorem.

$$
C=W \log _{2}(1+\mathrm{SNR})
$$

where $W$ is the bandwidth.

The theoretical BER for BPSK modulation over an additive white Gaussian noise (AWGN) channel is given by [11]

$$
P_{e}=\frac{1}{2} \operatorname{erfc}(\sqrt{\mathrm{SNR}}) .
$$

\subsection{System Parameters}

The performances of the MI wireless communication system can be estimated by the SNR as defined in the previous subsection, such as the expected transmission range and communication data rates under different distances.

To obtain high SNR on the receiver site, we choose coil antennas with high quality factor for MI wireless communications. Specifically, the coil antenna has 30 turns with inductance and resistance equal to $397 \mu \mathrm{H}$ and $3.7 \Omega$ respectively.

In our system design, the maximum exciting current on the transmitter side is $I_{0}=0.1 \mathrm{~A}$. This is mainly determined by the following facts. First, although increasing $I_{0}$ can increase the system performance, the improvement is very limited due to the fast attenuation of EM waves in seawater as shown in [15]. Second, it is complicated and expensive to design a small size power amplifier with higher output power that works on the spectrum from $100 \mathrm{kHz}$ to $1 \mathrm{MHz}$.

The noise voltage level was measured during the sea test.

\section{EXPERIMENT DESIGN}

The overview of the sea test is shown in Fig. 2. A remote operated vehicle (ROV) is used to carry a transmitter coil antenna, which will be submerged into the ocean at different depths. At the same time, a remote controlled boat (RCB) is used to carry a receiver coil which will also be submerged into the ocean. In this section, we will first introduce the ROV and RCB platforms, covering their relevant original specifications, modes of operation, and corresponding implementation of the modifications required for the experiments in this paper. Then, the hardware and software design of the MI wireless communication system is described.

\subsection{ROV \& RCB Platform}

The robotic system used for the experiment is the BlueROV2, a remotely operated vehicle (ROV) developed by Blue Robotics. The 


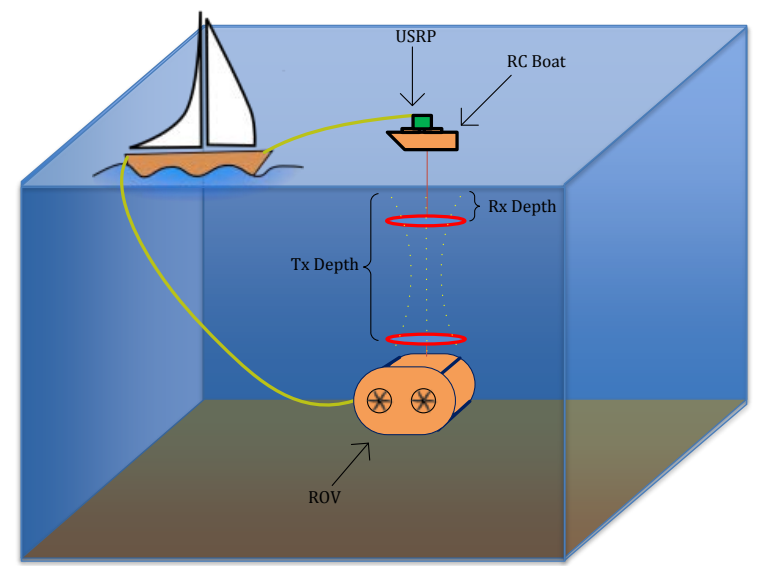

Figure 2: Overview of experiment setup. The ROV is stationed underneath the RC boat. As the ROV changes depth, the signal strength received by the boat varies. Both the boat and the ROV are tethered to laptop computers.

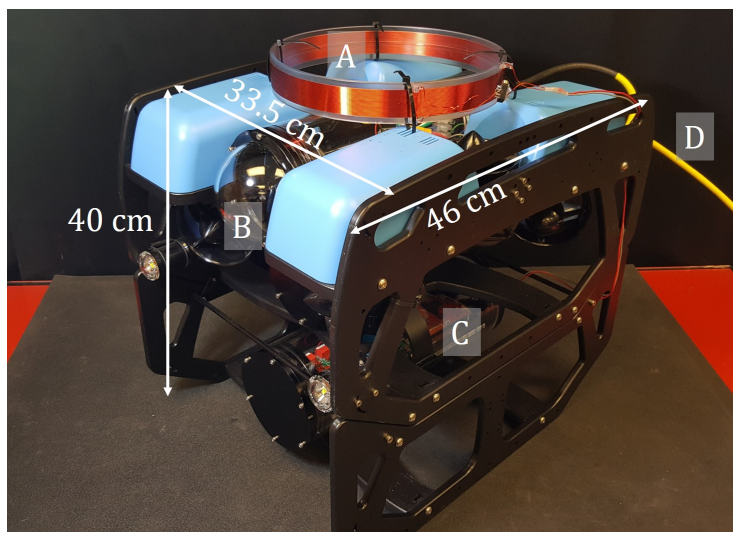

Figure 3: BlueROV2 with the underwater communication modifications. A) Transmitting coil. B) Main electronics enclosure. C) Underwater communication electronics enclosure. D) Tether to computer.

connection between the system and a computer is provided through a 150 meter long tether. The reconfigurable design of the BlueROV2 allowed us to add a payload skid, which held an additional 0.3 meter by 0.1 meter acrylic enclosure underneath the robot. This enclosure served as the waterproof housing for our communication electronics, which were connected to a coil antenna mounted on the top of the robot. The BlueROV2 with all attachments is shown in Fig. 3.

Remote operation of the vehicle is done through QGroundControl software, which allows a human operator to control the thrusters, lights, and servo motors that change the tilt of the camera, with the use of a gamepad. QGroundControl communicates with a Pixhawk flight controller within the ROV, that serves as the main computer, through the MavLink protocol. The Pixhawk is loaded with the Ardusub firmware, which was developed by Blue Robotics. The electronics for operation of the ROV are independent of the electronics for this underwater communication experiment.

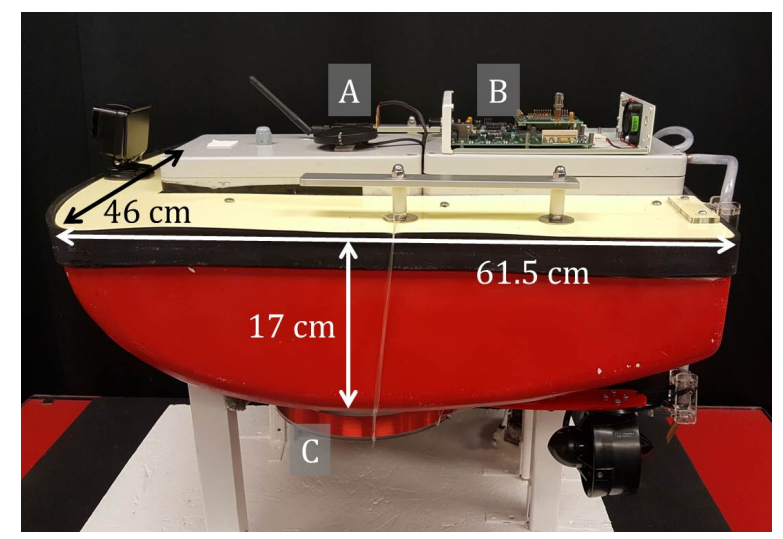

Figure 4: RCB with the underwater communication modifications. A) RTK GPS and radio antenna. B) USRP board for demodulation. C) Receiving coil.

The receiver antenna is connected to a remotely controlled boat (RCB), that has been modified to enable autonomous GPS waypoint following. A Pixhawk2.1 flight controller manages the boat, while real-time kinematic (RTK) GPS is provided by the HERE+ RTK GPS system. The coil antenna is mounted underneath the boat, and connected to a USRP for data analysis. Fig. 4 shows the boat with the USRP and antenna attached.

\subsection{Wireless Communication System}

By leveraging the Universal Software Radio Peripheral (USRP) and software-defined radio (SDR) technology, we can realize a prototype MI wireless communication system.

Due to size and waterproof issues, the USRP cannot be applied in underwater directly. In this work, we design a small size transmitter that can be carried by the ROV based on the Texas Instruments MSP432 microcontroller. The differential amplifier built on the high output current operation amplifier AD8615 was used to drive the transmission coil. The overall system setup was shown in Fig. 5.

The Texas Instruments MSP432P401R microcontroller was used to modulate the data for transmission. The program flow diagram is shown in Fig. 6. The modulated output is then sent to a signal amplifier to drive the coil antennas, which have compensating capacitors connected in serial. The measured inductance of the coils is $397 \mu \mathrm{H}$, necessitating a compensating capacitance of nearly $4 \mathrm{nF}$ to achieve resonance at the operating frequency of $125 \mathrm{kHz}$. The overall system power consumption is $0.3 \mathrm{~W}$.

\section{RESULTS \& DISCUSSIONS}

In this section, we first tested the performance of the MI wireless communication system in the air. Afterward, the system is tested in a pool. Finally, we traveled to La Porte, Texas, to carry out testing in seawater.

The experiment setup for the three different scenarios is shown in Fig. 7. We use the same transmitter as shown in Fig. 5 to drive a coil antenna. This will guarantee that the transmission power is unchanged. Similarly, we used the same receiver coil antenna for all scenarios. The only changing variable is the propagation medium, which includes air, fresh water, and sea water. 


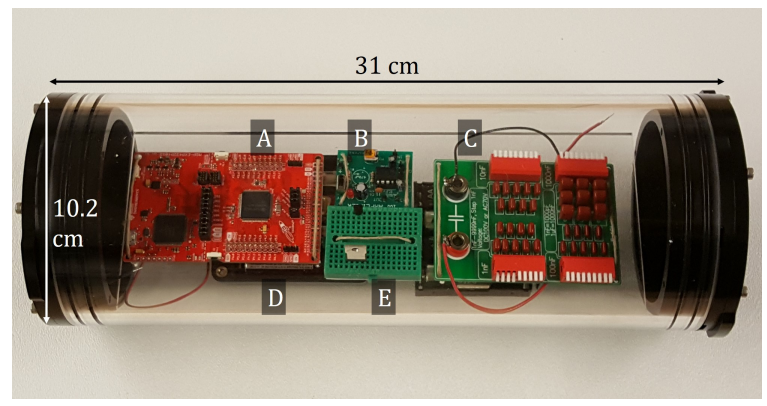

Figure 5: Underwater communication electronics inside a BlueROV2 enclosure. A) MSP432 microcontroller. B) Signal Amplifier. C) Compensating capacitor. D) Battery packs. E) Voltage regulating circuits.

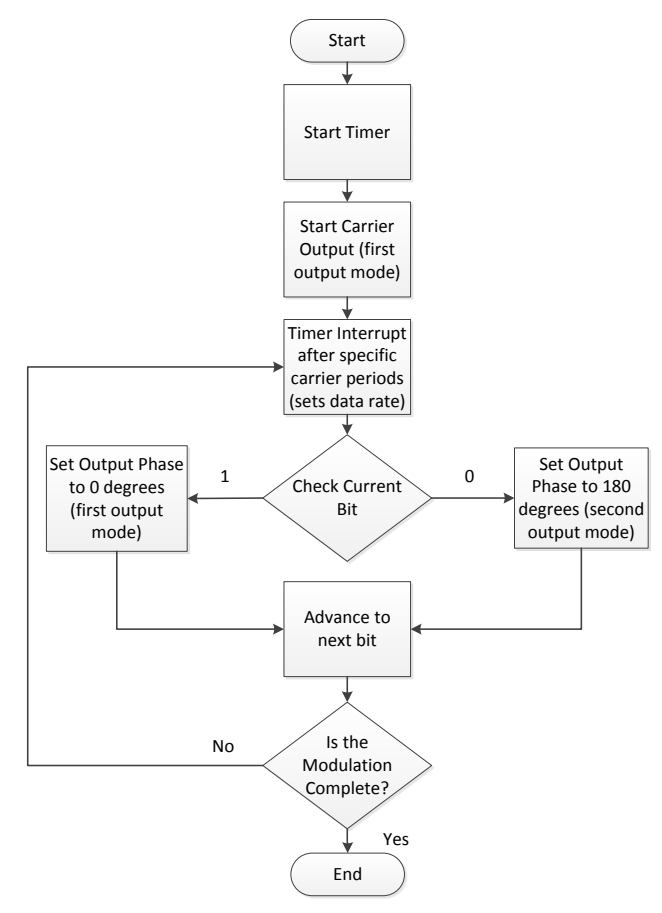

Figure 6: Flow diagram for BPSK modulation.

The pool test in particular was done by the proposed ROV \& $\mathrm{RCB}$ system. The transmitting coil was carried by the ROV and the receiving coil was attached to the bottom of the RCB as shown in Fig.7.b). Since the fresh water is clear, it is easier to align the two coil antennas by controlling the depth and position of the ROV.

Unfortunately, the proposed ROV \& RCB system is difficult to test in the sea water scenario, mostly due to the lack of visibility. Instead, the coil antennas were attached to tripods and covered with water proof bags to protect the electric components as shown in Fig.7.c). The tripods were then submerged into the sea water to a depth of $1 \mathrm{~m}$. The receiving coil was kept stationary while the separation between the two coils gradually increased. The signal strength on the receiving end was measured with the use of an oscilloscope, and then plotted against the separation distance.
The noise voltage level of the receiver circuit was around $15 \mathrm{mV}$, which is relatively high. The main reason for high noise level is that the majority of the system is connected through the use of a breadboard and jump wires. Even under such a less than ideal setup, the transmission range for MI can go as far as $3 \mathrm{~m}$ in air. The same MI signal attenuates much faster in fresh water, and it can only propagate up to $1 \mathrm{~m}$ in the sea. The testing results for the voltage measured at the receiving coil are shown in Fig. 8.

Based on Eq. 4 and Eq. 5, the estimated MI channel capacity in different propagation medium is shown in Fig. 9, while the estimated bit error rate is shown in Fig. 10. The bandwidth was set to be 60 $\mathrm{kHz}$.

\section{CONCLUSION \& FUTURE WORK}

In this paper we designed a Magnetic Induction wireless communication system, then performed tests on air, fresh water, and sea water. We measured the MI signal attenuation as a function of the separation distance between two coil antennas. The experiment incorporated the use of software defined radio and microcontrollers to transmit, receive, and analyze the data, in the case of the fresh water test, robotic platforms. Our results show that although MI signals attenuate at rapid rates in sea water, it still can propagate as far as $1 \mathrm{~m}$ with low power consumption and small coil antennas with radii equal to $10 \mathrm{~cm}$.

In the future, we will keep improving our ROV \& RCB platform to be able to carry out longer tests in more challenging environments. To be specific, we will integrate SDR technology into the ROV \& RCB, such that real time MI wireless communications can be realized between the robots. Then, the robots will be integrated with the Robot Operating System (ROS) to enable them to perform

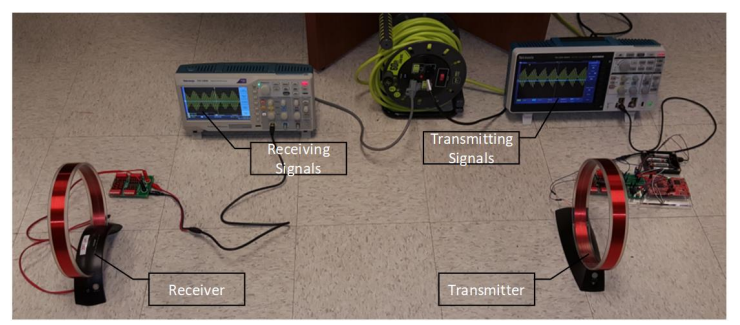

a)

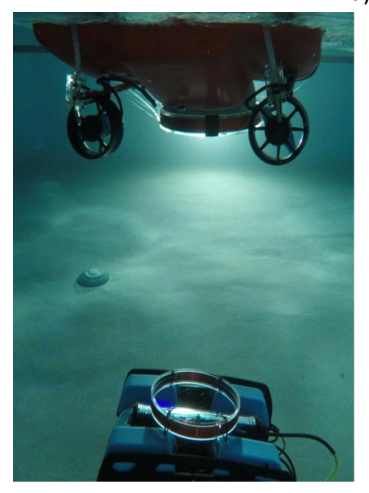

b)

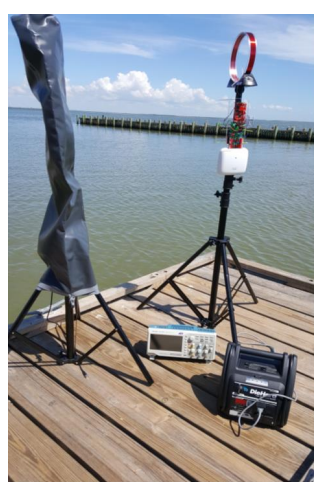

c)
Figure 7: System setup for MI testing. a): air test, b): pool test, c): sea test. 


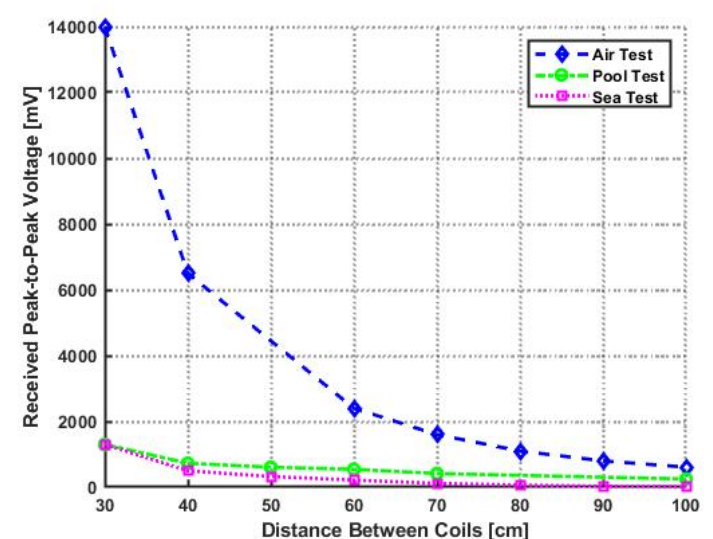

Figure 8: Voltage at the receiving coil as a function of the separation distance between the coil antennas.

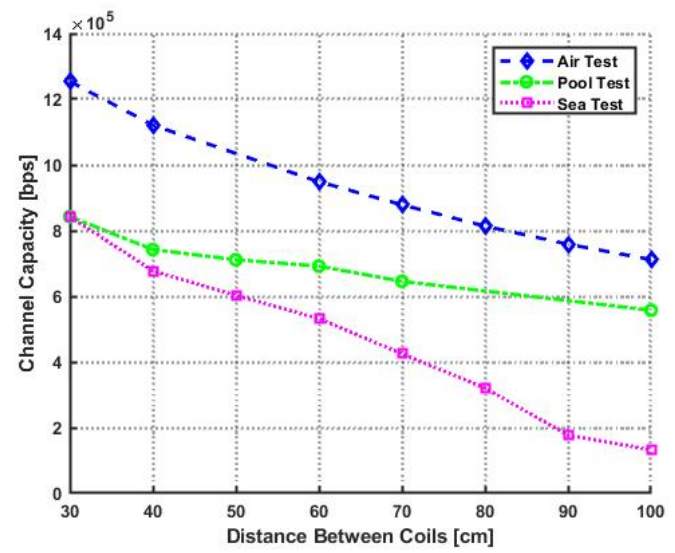

Figure 9: Channel Capacity as a function of the separation distance between the coil antennas.

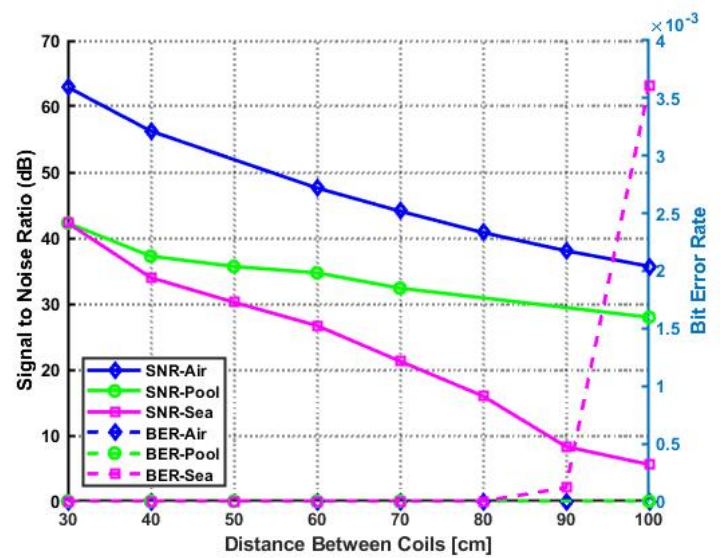

Figure 10: SNR and estimated BER as a function of the separation distance between the coil antennas.

various tasks autonomously, like utilizing computer vision, for example, to detect and establish communication with underwater sensor nodes and other systems without the need for a human operator. This will be particularly advantageous for precise experiments in low visibility environments.

Furthermore, we will measure the maximum communication range and bit error rate using BPSK, and QPSK modulations.

\section{ACKNOWLEDGEMENT}

This work was supported in part by the U.S. National Science Foundation under grants US CNS-1343361, CNS-1350230 (CAREER), CNS-1646607, CNS-1702850, CNS-1801925 and the Fundamental Research Funds for the Central Universities under Grant 2018XKJC03, China.

\section{REFERENCES}

[1] Niaz Ahmed, Justin Hoyt, Andriy Radchenko, David Pommerenke, and Y Rosa Zheng. 2014. A multi-coil magneto-inductive transceiver for low-cost wireless sensor networks. In Underwater Communications and Networking (UComms), 2014. IEEE, 1-5.

[2] Ian F Akyildiz, Pu Wang, and Zhi Sun. 2015. Realizing underwater communication through magnetic induction. IEEE Communications Magazine 53, 11 (2015), 42-48.

[3] Wei Cheng, Yu Luo, Zheng Peng, and Mardi C Hastings. 2014. A framework of acoustic channel availability prediction for avoiding interfering marine mammals. In Proceedings of the International Conference on Underwater Networks \& Systems. ACM, 34.

[4] Jun-Hong Cui, Jiejun Kong, Mario Gerla, and Shengli Zhou. 2006. The challenges of building mobile underwater wireless networks for aquatic applications. Ieee Network 20, 3 (2006), 12-18.

[5] Thuc Phi Duong and Jong-Wook Lee. 2011. Experimental results of high-efficiency resonant coupling wireless power transfer using a variable coupling method. IEEE Microwave and Wireless Components Letters 21, 8 (2011), 442-444.

[6] Hongzhi Guo, Zhi Sun, and Pu Wang. 2015. Channel modeling of mi underwater communication using tri-directional coil antenna. In Global Communications Conference, 2015 IEEE. IEEE, 1-6.

[7] Eugenio Jimenez, Gara Quintana, Pablo Mena, Pablo Dorta, Ivan Perez-Alvarez, Santiago Zazo, Marina Perez, and Eduardo Quevedo. 2016. Investigation on radio wave propagation in shallow seawater: Simulations and measurements. In Underwater Communications and Networking Conference (UComms), 2016 IEEE Third. IEEE, 1-5.

[8] Andre Kurs, Aristeidis Karalis, Robert Moffatt, John D Joannopoulos, Peter Fisher, and Marin Soljačić. 2007. Wireless power transfer via strongly coupled magnetic resonances. science 317, 5834 (2007), 83-86.

[9] Xuanheng Li, Yi Sun, Yuanxiong Guo, Xin Fu, and Miao Pan. 2017. Dolphins First: Dolphin-Aware Communications in Multi-Hop Underwater Cognitive Acoustic Networks. IEEE Trans. Wireless Communications 16, 4 (2017), 2043-2056.

[10] Yu Luo, Lina Pu, Michael Zuba, Zheng Peng, and Jun-Hong Cui. 2014. Cognitive acoustics: Making underwater communications environment-friendly. In Proceedings of the International Conference on Underwater Networks \& Systems. ACM, 48.

[11] Neha Mukul, Shailendra Singh Pawar, and Mohd Sarwar Raeen. 2014. BER and SER Based Performance Analysis of BPSK and QPSK Modulation Schemes with OFDM in Rayleigh Fading Channel. International fournal of Emerging Trends in Science and Technology 1, 08 (2014).

[12] Alanson P Sample, David T Meyer, and Joshua R Smith. 2011. Analysis, experimental results, and range adaptation of magnetically coupled resonators for wireless power transfer. IEEE Transactions on industrial electronics 58, 2 (2011), 544-554.

[13] Zhi Sun and Ian F Akyildiz. 2010. Magnetic induction communications for wireless underground sensor networks. IEEE Transactions on Antennas and Propagation 58, 7 (2010), 2426-2435.

[14] Yibin Wang, Andrew Dobbin, and Jean-François Bousquet. 2016. A compact low-power underwater magneto-inductive modem. In Proceedings of the 11th ACM International Conference on Underwater Networks \& Systems. ACM, 14.

[15] Debing Wei, Li Yan, Xuanheng Li, Yi Sun, Dongfeng Yuan, Jiefu Chen, and Miao Pan. 2018. Exploiting Magnetic Field Analysis to Characterize MI Wireless Communications in Subsea Environments. In 2018 International Conference on Computing, Networking and Communications (ICNC). IEEE, 805-809.

[16] Debing Wei, Li Yan, Xunheng Li, Jie Wang, Jiefu Cheng, Miao Pan, and Yahong Zheng. 2018. Ferrite Assisted Geometry-Conformal Magnetic Induction Antenna and Subsea Communications for AUVs. In Global Communications Conference (GLOBECOM), 2018 IEEE. IEEE, 1-6. 